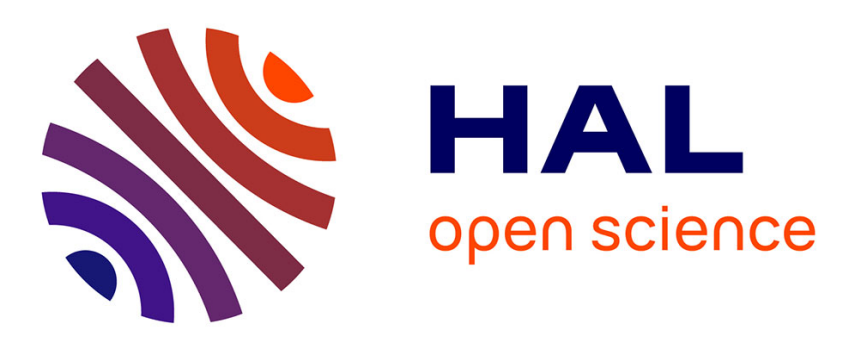

\title{
Une écriture littéraire de la généalogie ? Jean-Baptiste L'Hermite de Soliers
}

\author{
Marine Roussillon
}

\section{To cite this version:}

Marine Roussillon. Une écriture littéraire de la généalogie ? Jean-Baptiste L'Hermite de Soliers. Dix-septième siècle, 2020, nº288 (3), pp.485-496. 10.3917/dss.203.0485 . hal-03006899

\section{HAL Id: hal-03006899 \\ https://hal.science/hal-03006899}

Submitted on 14 Feb 2022

HAL is a multi-disciplinary open access archive for the deposit and dissemination of scientific research documents, whether they are published or not. The documents may come from teaching and research institutions in France or abroad, or from public or private research centers.
L'archive ouverte pluridisciplinaire HAL, est destinée au dépôt et à la diffusion de documents scientifiques de niveau recherche, publiés ou non, émanant des établissements d'enseignement et de recherche français ou étrangers, des laboratoires publics ou privés. 


\section{Une écriture littéraire de la généalogie ? Jean-Baptiste L'Hermite de Soliers}

« On devrait, dans une république bien ordonnée, défendre d'écrire à des gens faits comme cela ». C'est ainsi que Samuel Guichenon, historiographe de la cour de Savoie et généalogiste reconnu, parle de son confrère Jean-Baptiste L'Hermite de Soliers, seigneur de Vauselle, lui aussi auteur d'une douzaine de traités généalogiques entre 1655 et 1669. L'Hermite est accusé d'imposture et de vénalité : il vendrait aux plus offrants des généalogies flatteuses. Il est vrai que contrairement à Guichenon ou aux autres généalogistes patentés de la deuxième moitié du XvII ${ }^{\mathrm{e}}$ siècle, L'Hermite n'occupe aucune fonction officielle, ni ne joue aucun rôle dans le contrôle de la noblesse par la monarchie. Il est d'abord un homme de lettres qui cherche à vivre de sa plume, auteur d'une tragédie à machines et de quelques vers, frère du poète Tristan L'Hermite, et allié à par sa femme à plusieurs comédiens de la troupe de Molière.

La production généalogique d'un tel littérateur peut sembler marginale au regard du grand récit qui fait de l'essor de la généalogie au $\mathrm{XVII}^{\mathrm{e}}$ siècle une étape dans la mise en œuvre d'une histoire scientifique $^{1}$. Elle met pourtant en lumière la continuité entre les écrits généalogiques et d'autres écrits que nous avons l'habitude de considérer comme « littéraires » : la tragédie, le ballet, les vers d'éloge, le roman à clé... Elle rend ainsi visibles des usages sociaux de la généalogie qui ne reposent ni sur son efficacité juridique, ni sur sa vérité historique ${ }^{2}$, mais plutôt sur sa capacité à représenter des identités ou des pouvoirs, à susciter le plaisir et l'admiration.

L'étude de ces usages invite à reconsidérer le clivage entre histoire et fiction. Ce clivage, évident pour nous, apparaît au $\mathrm{XVII}^{\mathrm{e}}$ siècle comme une construction polémique dont les enjeux sont à la fois éthiques et politiques. Accuser L'Hermite d'imposture est une manière de disqualifier une écriture non-scientifique du passé, mais c'est aussi un moyen d'affirmer le monopole du pouvoir monarchique sur la définition de la noblesse ${ }^{3}$ : condamner la vénalité de son écriture, n'est-ce pas dire que le seul discours vrai sur l'ordre social, c'est celui que tient (ou commande) le pouvoir monarchique ? Étudier la production généalogique d'un homme de lettres conduit à défaire le lien privilégié entre généalogie et histoire, à interroger le clivage entre histoire et fiction, pour mettre en lumière le processus conflictuel de définition de ce qui fonde la valeur des écrits.

Du théÂtre À LA GÉNÉALOGIE : UNE VUE D’ENSEMBLE

Genres à la mode et formes de l'éloge

\footnotetext{
$1 \quad$ Voir Olivier Poncet, « Cercles savants et pratique généalogique en France (fin XVI ${ }^{\mathrm{e}}$-milieu du XVII ${ }^{\mathrm{e}}$ siècle) », in Olivier Rouchon (dir.), L’Opération généalogique. Cultures et pratiques européennes, $x V^{e}-X_{V I I I}{ }^{e}$ siècles, Rennes, P.U. Rennes, 2014, pp. 101-136.

${ }^{2}$ Sur ces pratiques généalogiques qui échappent à notre conception de la vérité historique, voir Roberto Bizzocchi, Généalogies fabuleuses. Inventer et faire croire dans l'époque moderne [1995], trad. fr. Paris, Éditions rue d'Ulm, 2010. Je me permets aussi de renvoyer à mon article, «Un roman de chevalerie en guise de généalogie. Le chevalier Aymon de Salvaing de Boissieu et les écrivains du Dauphiné », in Isabelle Luciani et Valérie Pietri, L'incorporation des ancêtres. Généalogie, construction du présent. Du Moyen-âge à nos jours, Presses Universitaires de Provence, 2016, pp. 89-101.

Voir Dinah Ribard, «Travail intellectuel et violence politique : théoriser la noblesse en France à la fin du XVIIe siècle ", in Vincent Azoulay et Patrick Boucheron (dir.), Le Mot qui tue. Une histoire des violences intellectuelles de l'Antiquité à nos jours, Seyssel, Champ Vallon, 2009, pp. 353-368.
} 
Dans les premières années de sa carrière, les tentatives de Jean-Baptiste l'Hermite pour vivre de son écriture passent par la pratique de genres à la mode, susceptibles de lui apporter le succès : en 1639 il publie une tragédie à machines, La Chute de Phaeton, puis en 1645 il exploite la vogue des vies de femmes illustres avec La Princesse hérö̈que ${ }^{4}$. Cette quête de succès s'appuie sur les réussites de son frère, le poète Tristan L'Hermite. La Chute de Phaeton paraît au moment où Tristan triomphe au théâtre avec sa Mariane. En 1650, quand Tristan est reconnu comme poète, Jean-Baptiste publie à son tour des vers, un Mélange de poésies hérö̈ques et burlesques peut-être écrit en collaboration avec son frère 5 .

Parallèlement, il cherche à s'inscrire dans des relations de clientèle. La Chute de Phaeton est dédiée à Esprit de Rémond, seigneur de Modène, ancien page de Gaston d'Orléans aux côtés de Tristan L'Hermite et ami du duc de Guise ${ }^{6}$. La Princesse hérö̈que s'achève sur un tableau généalogique des descendants de la princesse ${ }^{7}$, dont la dernière ligne est consacrée aux ducs de Guise. L'écriture généalogique apparaît ici comme une forme de l'écriture encomiastique.

La même année 1645 voit paraître le premier ouvrage explicitement généalogique de Jean-Baptiste L'Hermite : ce sont les Éloges de tous les premiers présidents du Parlement de Paris, avec leurs généalogies, écrits en collaboration avec François Blanchard, publiés aux dépens des auteurs et vendu chez Cardin Besongne. L'écriture généalogique de Jean-Baptiste L'Hermite n'y est pas une écriture savante. Si l'on en croit son collaborateur, L'Hermite a joué le rôle d'un simple intermédiaire de publication - peu scrupuleux de surcroît :

Le petit Traicté des premiers Presidens qui fut donné au public il y a deux ans, [a] esté assez bien reçeu, bien que la facilité de celuy à qui j'avois confié mes Memoires, et qui l'a fait imprimer sous son nom conjoinctement avec le mien, y eust alteré plusieurs choses et inseré quelques généalogies mal justifiées ${ }^{8}$.

Dans la pratique de Jean-Baptiste L'Hermite, la généalogie apparaît ainsi à la fois comme un genre à la mode, au même titre que la tragédie à machines ou les vies de femmes illustres, et comme une forme de l'écriture d'éloge capable de susciter des protecteurs.

\section{Comédien et généalogiste}

Auteur de théâtre, Jean-Baptiste L'Hermite fut aussi, brièvement, comédien. Lié à la troupe de Molière par son protecteur, le seigneur de Modène, amant de Madeleine Béjart, et par sa femme, Marie Courtin, une tante de la comédienne, il la rejoint au début des années $1650^{9}$, quand elle joue dans le sud de la France.

À la même période, Joseph Béjart, le frère aîné de Madeleine, lui aussi comédien de Molière, publie coup sur coup un Recueil des tiltres, qualités, blazons et armes des seigneurs barons et des seigneurs prélats des Estats généraux de la province de Languedoc dédié au prince de Conti en 1654 et un Recueil des qualitez, armes, blasons des États généraux tenus à Pézenas en 1655, encore dédié à Conti et pour lequel l'assemblée des États de Languedoc lui accorde une gratification de 1500 livres. Les deux livres sont richement illustrés. Ils commémorent les États de Languedoc pour lesquels la troupe a été appelée à jouer, et à l'occasion desquels elle est passée sous la protection du

$4 \quad$ Paris, Cardin Besongne, 1639 ; et Paris, Cardin Besongne, 1645. Ce dernier titre concurrence directement $L a$ femme héroïque du père Du Bosc, qui est publié la même année chez Antoine de Sommaville et Augustin Courbé.

Les deux volumes des Femmes illustres de M. de Scudéry ont paru en 1642 et 1644 chez les mêmes.

$5 \quad$ Voir Laurence Grove, « Les Poésies hérö̈ques et burlesques (1650) : Jean-Baptiste et/ou Tristan ? », Cahiers

Tristan L'Hermite XXIV : Le Quatrième Centenaire, 2002, pp. 77-97.

6 Sur ce personnage et ses liens avec Madeleine Béjart, voir Henri Chardon, Nouveaux Documents sur la vie de Molière. M. de Modène, ses deux femmes et Madeleine Béjart, Paris, Picard, 1886.

Op. cit., n.p.

Paris, Cardin Besongne, 1647, Préface, n.p.

Il joue notamment un petit rôle dans les représentations d'Andromède données à Lyon en 1653. Sa femme et sa fille y participent aussi. Voir Georges Forestier, Molière, Paris, Gallimard, 2018, pp. 73-78 et p. 92 et Napoléon-Maurice Bernardin, Un précurseur de Racine. Tristan L'Hermite, sieur du Solier (1601-1655). Sa famille, sa vie, ses œuvres [1895], Genève, Slatkine, 1967, p. 285-286. Sur l'alliance entre le frère de Tristan L'Hermite et la famille Béjart, voir Claire Chatelain, « De l'épée à la plume : l'itinéraire d'un noble déclassé mais non disgracié, Tristan l'Hermite », dans Mathilde Bombart (dir.), Lectures de Tristan L'Hermite, P.U. Rennes, 2013, p. 29-47, p. 40-41. 
prince de Conti. La continuité entre la production de spectacles et celle de ces livres est manifeste : l'une comme l'autre sont inscrites dans une relation de service; il s'agit de publier la gloire du commanditaire et de plaire à l'élite locale.

En outre, les deux ouvrages donnent une représentation ordonnée de la noblesse de Languedoc qui les rapprochent d'un spectacle auquel Molière et Joseph Béjart participent en 1655 : le Ballet des Incompatibles $^{10}$. Les recueils d'armoiries, comme le ballet, contribuent à configurer les hiérarchies locales au lendemain de la Fronde.

Le cas de Joseph Béjart met en lumière le caractère anachronique de l'opposition entre une écriture généalogique savante, sérieuse, et une activité de comédien qui relèverait du divertissement. L'activité théâtrale et l'écriture généalogique relèvent d'un même ensemble de pratiques : des pratiques inscrites dans des relations de clientélisme, dont l'objet est la publication et la configuration d'un ordre social.

\section{La spécialisation généalogique}

C'est surtout après la mort de son frère que Jean-Baptiste L'Hermite se spécialise dans les généalogies avec notamment une série de traités sur « les Estrangers françois, contenant les éloges, armes et blasons des plus illustres personnages sortis d'Italie, Allemagne et autres pays, lesquels ont esté affectionné à cette Couronne [de France] ${ }^{11}$. Il publie à Arles, chez François Mesnier, successivement La Toscane Française (1657) et La Ligurie Française (1659), ainsi que Les Présidents nés des Estats de la province de Languedoc ou Cronologie des archevesques et primats de Narbonne, contenant les éloges desdits prélats, ensemble leurs armes, blazonnées et gravées en taille douce (1659). En 1658, à l'occasion de l'entrée de Louis XIV à Lyon, il fait imprimer à ses dépens dans cette ville un ouvrage intitulé Les forces du Lyon, ou les Armoiries des Capitaines, Lieutenants et enseignes des personnages de la Ville de Lyon, qu'il tente de vendre aux principales familles de la ville ${ }^{12}$. Puis au début des années 1660 il reprend la série des «Étrangers François », cette fois-ci chez des libraires parisiens : La France espagnole (vers 1662), qui fait suite au mariage de Louis XIV, puis de nouveaux ouvrages sur les familles italiennes alliées à la France, Naples française (1663) et Les Corses français (1667). Son dernier ouvrage généalogique est l'Histoire généalogique de la noblesse de Touraine, dont le privilège est accordé en 1665 mais dont l'achevé d'imprimer date de 1667. Tous ces traités sont de grand format et abondamment illustrés.

Les privilèges et pages de titre de ces traités présentent l'auteur comme un « chevalier de l'Ordre du Roi, et l'un des Gentils-hommes servans de sa Majesté ${ }^{13}$. La revendication de proximité avec le roi et la cour accrédite le discours généalogique. Comme dans ses premiers ouvrages, Jean-Baptiste L'Hermite cherche aussi à utiliser la réputation littéraire de son frère pour assurer le succès de ses propres productions. Sur les pages de titre des traités, l'auteur est tantôt désigné comme « JeanBaptiste L'Hermite, dit Tristan $\|^{14}$ tantôt comme "Jean-Baptiste Tristan L'Hermite ${ }^{15}$ tantôt même comme "Tristan L'Hermite $»^{16}$. Cette appropriation du pseudonyme de son frère fait le pari que la réputation acquise dans l'écriture de vers ou de tragédies peut être réinvestie dans l'écriture généalogique.

La spécialisation généalogique ne fait pas pour autant de Jean-Baptiste L'Hermite un érudit. Sa production, si elle adopte les formes d'une écriture savante, se fonde essentiellement sur la reproduction des documents fournis par les familles, sur des emprunts massifs aux ouvrages

10 Philippe Mauran, « Le Ballet des Incompatibles (Montpellier-1655) ou l'état du Languedoc en 1655 », Dixseptième siècle, 2014/4 ( $\left.\mathrm{n}^{\circ} 265\right)$, pp. 691-707.

11 Ce sont les termes utilisés dans le privilège d'ensemble accordé en 1656. Voir J.-B. L'Hermite, La Toscane françoise, Arles, F. Mesnier, 1657, n.p.

12 D'après une lettre de Samuel Guichenon à Antoine de Ruffi citée par H. Chardon, Nouveaux Documents sur la vie de Molière. M. de Modène, ses deux femmes et Madeleine Béjart, Paris, Picard, 1886, p. 387.

13 La Toscane françoise, Arles, Tristan Mesnier, 1658.

14 Dans Naples françoise, Paris : S. Martin et P. Bienfait, 1663 ou dans Italie françoise, Rouen, L. Maurry, 1664.

15 Dans le Discours généalogique de la maison del Bene, Paris, 1662.

16 Dans La Ligurie françoise, Avignon, imp. de G. Brandreau, 1659. 
généalogiques publiés par ailleurs, et sur le recyclage de ses propres productions. La Naples française est republiée dès 1664 sous le titre L'Italie française et plusieurs généalogies particulières extraites de ces traités font l'objet de publications séparées (Discours historique et généalogique sur l'illustre maison de Mancini, 1661, Discours généalogique de la maison del Bene, 1662). Il s'agit de s'inscrire dans la mode du traité généalogique pour en tirer profit, de diverses manières. D'une part, ces ouvrages permettent de vendre aux familles un objet de magnificence (par sa forme) qui légitime aussi leur pouvoir par son contenu. D'autre part, ils s'inscrivent dans des relations de clientèle. L'intérêt pour l'Italie est lié à la fois à la guerre qui s'y déroule ${ }^{17}$ et au rôle qu'y a joué le seigneur de Modène, qui a suivi le duc de Guise dans son expédition à Naples et qui publie en 1665 une Histoire des révolutions de Naples. Enfin, ces ouvrages attribués à un poète et tragédien renommé peuvent intéresser un public mondain par leur forme agréable. La pratique de JeanBaptiste L'Hermite met ainsi en lumière un usage de l'histoire généalogique comme genre à la mode, disponible pour un écrivain qui cherche à vivre de sa plume, et susceptible de plaire indépendamment de sa valeur historique.

\section{Auto-généalogies}

En 1667, au moment où paraît l'Histoire généalogique de la noblesse de Touraine, Jean-Baptiste L'Hermite fait aussi paraître une nouvelle édition du roman de son frère, Le Page disgracié, qu'il accompagne d'une clé censée en identifier les principaux personnages ${ }^{18}$. Dès la première « remarque », cette clé propose une généalogie des L'Hermite. La phrase « Je suis sorti d'une assez bonne maison » est abondamment glosée dans une généalogie ascendante qui part de Tristan et de ses parents, "Pierre L'Hermite, Chevalier Seigneur de Souliers et d'Élisabeth Miron» pour remonter aux «fondateurs de [cette] maison »: « les anciens Comtes de Clermont d'Auvergne, puînés des princes souverains comtes d'Auvergne » et "Pierre L'Hermite, auteur de la première croisade ». La seconde remarque complète cette généalogie en évoquant notamment Tristan L'Hermite, grand prévôt de Louis $\mathrm{XI}^{19}$. Là encore, la distinction entre un roman relevant de la fiction et du divertissement et une clé qui proposerait un discours savant et référentiel ne tient pas : la généalogie proposée par les remarques est largement mythique. La clé et le roman apparaissent comme deux formes de l'écriture de soi ${ }^{20}$.

Le mythe familial développé dans la clé du Page disgracié est déjà présent dans les ouvrages antérieurs de Jean-Baptiste L'Hermite. Dans La Princesse héroïque, le récit de la première croisade est prétexte à évoquer la descendance de Pierre l'Ermite et une note marginale rattache cette généalogie à la famille de l'auteur : "Pierre l'Hermite premier du nom glorieux en sa postérité, renommée en France et en Espagne sous le nom de 1'Hermite de Souliers $»^{21}$. L'année même où paraît La Princesse héroïque, en 1645, Jean-Baptiste L'Hermite donne aussi (de manière anonyme) une nouvelle édition de La Vie du vénérable Pierre l'Hermite du jésuite Pierre d'Outreman, parue pour la première fois en 1632. Le texte de la première édition est modifié en plusieurs endroits pour construire un lignage continu liant Pierre l'Ermite, Tristan l'Hermite, le grand prévôt de Louis XI, et la famille L'Hermite de Soliers dont sont issus Tristan et Jean-Baptiste ${ }^{22}$. Ces ancêtres mythiques sont ensuite convoqués dans les ouvrages de Jean-Baptiste L'Hermite pour définir une identité d'auteur, à la fois noble et littéraire. Dans les Mélanges de poésies hérö̈ques et burlesques, les vers

17 Voir Anna Blum, La Diplomatie de la France en Italie du Nord au temps de Richelieu et de Mazarin, Paris, Classiques Garnier, 2014.

18 Tristan L'Hermite, Le Page disgracié, Paris, A. Boutonné, 1667.

19 Jean-Baptiste L'Hermite, « Clef du Page disgracié » dans Tristan L'Hermite, Euvres complètes, Paris, Champion, 1999, I, p. 418 et 419-420.

20 Sur cette clé comme forme de l'écriture de soi, voir Mathilde Bombart, « Roman personnel ou roman familial ? Autour de la clef du Page disgracié », actes du colloque Actualités de Tristan l'Hermite, Université de Nanterre/E.N.S. Ulm, novembre 2001, Littérales, numéro spécial Actualité de Tristan, 2003, éd. Jacques Prévot, p. 195-210.

$21 \quad$ J ;-B. L'Hermite, La Princesse hérö̈que, op. cit., p. 94

22 P. D'Outreman, La Vie du Vénérable Pierre L'Ermite..., Paris, L. Boulanger, 1645, p. 143-145. Sur cette réédition, voir N.-M. Bernardin, op. cit., p. 7 et p. 10-11. Cette revendication généalogique n'a aucun fondement historique. 
placés sous le portrait de l'auteur revendiquent l'héritage de Pierre l'Ermite. Dans Les Présidents nés des Estats de la Province du Languedoc, l'auteur se présente comme descendant « des anciens comtes de Clermont Auvergne ». Dans l'Histoire généalogique de la noblesse de Touraine, il s'approprie la devise de Tristan l'Hermite, grand prévôt de Louis XI - « Prier vaut à l'hermite » - et l'illustre de gravures représentant la première croisade, faisant la synthèse des deux ancêtres mythiques.

Cette vue d'ensemble des écritures généalogiques de Jean-Baptiste L'Hermite permet de formuler une série d'hypothèses.

La généalogie y apparaît comme une pratique polymorphe, circulant dans des genres divers et adoptant des formes variées. Elle se définit comme une écriture de service, destinée à la fois à des élites locales et à des figures de protecteurs. D'un côté, l'écrivain offre aux notables locaux la possibilité de publier et de légitimer leur position sociale - offre particulièrement intéressante au lendemain de la Fronde, dans un moment de reconfiguration des élites. De l'autre, elle publie la gloire de celui à qui est adressé le livre. Ces usages de l'écriture généalogique sont déjà bien repérés, mais la pratique de Jean-Baptiste L'Hermite met en lumière la parenté (à la fois circonstancielle et formelle) qu'ils entretiennent avec d'autres formes de représentation du pouvoir, comme le ballet ou le livre d'entrée, et avec d'autres écritures inscrites dans des relations de clientèle, notamment l'écriture de vers et l'écriture théâtrale.

Les tentatives de Jean-Baptiste L'Hermite de s'appuyer sur la réputation littéraire de son frère pour faire valoir son entreprise généalogique donnent un sens nouveau à ces voisinages. Le nom de Tristan ne tient en effet sa valeur ni de sa position sociale, ni d'un savoir spécifique qui lui serait associé, mais bien de son statut d'écrivain. En convoquant ce nom - dont il faut rappeler qu'il est un pseudonyme, un «nom d'auteur»-pour autoriser ses traités généalogiques, Jean-Baptiste L'Hermite s'appuie sur le processus d'institution de la littérature (de transformation des pratiques littéraires en valeur) ${ }^{23}$ pour donner cette même valeur à ses propres écrits.

\section{L'HISTOIRE GÉNÉALOGIQUE DE LA NOBLESSE DE TOURAINE : ÉCRIRE AVEC LA LitTÉRATURE}

Mettons ces hypothèses à l'épreuve d'une lecture plus rapprochée du dernier ouvrage généalogique de Jean-Baptiste L'Hermite: L'Histoire généalogique de la noblesse de Touraine ${ }^{24}$. Le titre revendique l'appartenance de l'ouvrage au genre savant de l'histoire généalogique, alors en pleine expansion, et une inscription locale dans la province de Touraine.

\section{La dédicace à Saint-Aignan}

Le livre est logiquement dédié au gouverneur de la région, François de Beauvilliers, duc de SaintAignan et premier gentilhomme de la chambre du roi. Or Saint-Aignan est aussi un ancien protecteur de Tristan L'Hermite. Ce choix sert ainsi à convoquer dans l'ouvrage une autorité à la fois familiale et littéraire qui éclaire certains choix d'écriture.

Saint-Aignan fut gouverneur de Touraine de 1661 à 1667. Lorsque l'ouvrage paraît, en 1667, il a cédé son gouvernement à Philippe de Courcillon de Dangeau. L'avant-propos mentionne d'ailleurs « Monsieur le marquis d'Angeau [sic], aujourd'hui gouverneur de la dite Province » (n.p.). Le choix de maintenir la dédicace à Saint-Aignan malgré ce changement est révélateur : c'est la relation de

23 Sur ce processus, voir AlainViala, Naissance de l'écrivain. Sociologie de la littérature à l'âge classique, Paris, Éditions de Minuit, 1985 et «L'Histoire des institutions littéraires », dans L'Histoire littéraire aujourd'hui, sous la direction d'Henri Béhar et Roger Fayolle, Paris, Armand Colin, 1990, pp. 118-128 ; ainsi que Christian Jouhaud, Les Pouvoirs de la littérature. Histoire d'un paradoxe. Paris, Gallimard, 2000.

24 Histoire généalogique de Touraine, enrichie des armes en taille-douce de chaque famille et de plusieurs portraits des plus illustres qui en sont sortis. Le tout tiré sur les originaux des chartes, titres et trésors des maisons ..., Paris, J. Langlois, Fr. Clouzier et Jacques Langlois fils, 1665. Sur ce traité, voir V. Pietri, « Les nobiliaires provinciaux et l'enjeu des généalogies collectives en France, XVIIe-XVIIIe s. », dans O. Rouchon (dir.), op. p. 216. 
clientèle qui a dicté le choix de l'objet traité, et non l'inverse. La dédicace désigne Saint-Aignan comme un protecteur de la « famille » de l'auteur :

Monseigneur,

Depuis que nostre famille fait son bouclier de l'honneur de vostre protection, que vos graces se sont respandues jusques à moi, et qu'après la mort de mon frère vous daignez encore par une enchaisnure continuelle de vos faveurs m'attacher au nombre de vos creatures; Le souvenir de tant d'obligations, MONSEIGNEUR, avoit toûjours estonné la feblesse de ma reconnoissance. (n.p.)

Saint-Aignan est en effet un ancien protecteur du poète Tristan L'Hermite ${ }^{25}$. À la fin de la dédicace, Jean-Baptiste L'Hermite rappelle cette relation en citant les vers écrits par son frère à la gloire du dédicataire :

C'est ce que promettoient les fleurs du Printemps de vos années, et les judicieuses prédictions qui faisoient autrefois parler ainsi mon frère :

Tu ne doit [sic] mourir ce dit-on

Qu'apres vingt conduittes d'armées,

Appuyé dessus un baston,

Où les fleurs de Lys sont semées. (n.p.) ${ }^{26}$

Dédier l'Histoire généalogique de Touraine à Saint-Aignan, c'est chercher à réactiver une relation ancienne, en la déplaçant de Tristan à sa famille, et donc à son frère. C'était déjà l'objet de la Naples françoise, publiée en 1663 et dédiée à Saint-Aignan. Cette première dédicace semble avoir eu une certaine efficacité, si l'on en croit les remerciements formulés ici. Avec l'Histoire généalogique de Touraine, il s'agit de réitérer l'opération, voire d'inscrire la relation de clientèle dans la durée. Comme dans l'édition du Page disgracié publiée la même année, l'écriture généalogique sert à la gestion d'un patrimoine littéraire et familial.

La citation des vers de Tristan est immédiatement suivie d'un éloge du dédicataire qui le présente comme un protecteur des lettres :

J'ajouste à ces héroïques qualitez, l'accompagnement des graces qui vous sont si naturelles, et qui se meslent si agréablement à la grandeur de vostre courage ; Les charmes dont vous engagez les volontez, et cette douce necessitez que vous imposez à tous ceux qui vous approchent, de vous aimer et de vous craindre. Les belles lumières dont vous éclairez le Parnasse, ces hautes élevations d'esprit qui vous font regner si souverainement entre nos Muses...

Dans les années 1660, Saint-Aignan a en effet la réputation de protéger les gens de lettres. En 1663, à l'occasion de son entrée à l'Académie française, la Muze historique de Loret le désigne comme « un des plus polis de la cour » qui « chérit les gens de savoir et d'esprit/ dont sa belle âme est vraie amie $\aleph^{27}$. Il est responsable, en tant que premier gentilhomme de la chambre du Roi, de l'organisation des divertissements de la cour, ce qui en fait un intermédiaire privilégié du mécénat royal. Il s'est vu dédier plusieurs ouvrages de théâtre : le Fantôme amoureux de Quinault (1658), la tragédie Nitétis de Desjardins (1663), la Thébaïde de Racine (1664). En 1664, il a aussi donné une pièce à la troupe de Molière (Bradamante ridicule), avant d'inviter cette troupe à jouer dans la première fête organisée par Louis XIV à Versailles. Si la dédicace de l'Histoire généalogique de Touraine ne mentionne pas les liens de Jean-Baptiste L'Hermite avec les Béjart, on peut supposer que le choix du dédicataire s'appuie aussi sur cette alliance. La dédicace place ainsi l'Histoire généalogique de Touraine dans la continuité d'une série d'autres écrits : les vers de Tristan, les pièces de théâtre dédiées à Saint-Aignan ou écrites par lui.

\section{Une identité familiale : figures d'écrivains et imaginaire romanesque}

La référence à Tristan apparaît à nouveau à la fin du traité, dans le passage des " additions » consacré à la famille de Chasteau-Châlon. La généalogie de cette grande famille tourangelle

$25 \quad$ Tristan publie un échange avec Saint-Aignan dans ses Lettres meslées (1642) et lui dédie sa Mort du Sage en 1644 et ses Vers héroïques en 1648.

26 Les vers de Tristan L'Hermite sont issus des Vers hérö̈ques, LX, p. 228, v. 33-36.

27 Loret, La Muze historique, Paris, Jannet, Daffis et Champion, t. 4, p. 83. 
s'achève sur l'évocation de la grand-mère maternelle de l'auteur, Denise de Saint-Prés, et de sa descendance :

De ce mariage ne sortit qu'une fille,

Denyse de S. Prez, femme de Pierre Miron Chevalier, Baron de Cramail, Gouverneur et Bailly de Chartres ; de laquelle alliance sont issues plusieurs filles, entre lesquelles

Elizabeth Miron, femme de Pierre l'Hermite, Chevalier Seigneur de Souliers, Gouverneur du Fort de la Chapelle Taillefer, père et mère de plusieurs enfans, entre lesquels

Tristan l'Hermite, Chevalier Seigneur de Souliers, Gentilhomme de la suite de feu Monseigneur le Duc d'Orléans, renommé par les excellentes Poësies qu'il a mises au jour.

Severin l'Hermite, tué au service du Roi devant la ville de Royan.

Jean-Baptiste l'Hermite, Chevalier, Gentil-homme Servant du Roi, dit le Chevalier de l'Hermite, Autheur de ce present Ouvrage ; lequel de son Mariage avec Marie Courtin de la Dehors, a une fille unique,

Magdelaine l'Hermite de Souliers, femme de Messire Esprit de Raymond, Comte de Modène, cy-devant Chambellan de feu Monseigneur le Duc d'Orléans et depuis Mestre de Camp general de la Ville et du Royaume de Naples, sous l'authorité de la Republique ${ }^{28}$.

Une fois de plus, la généalogie articule identité familiale et identité d'auteur : les mentions de Tristan et de Jean-Baptiste insistent sur leur activité d'écriture ; Tristan est nommé par son nom d'auteur, et pas par son véritable prénom (François) ; et les trois membres de la fratrie nommés sont précisément ceux qui figurent dans l'édition du Page disgracié parue la même année. Les dernières lignes de la généalogie permettent en outre de publier le mariage de la fille de Jean-Baptiste avec son ancien protecteur, Esprit de Raymond seigneur de Modène, et de revendiquer pour l'auteur un statut social élevé.

Cette généalogie n'occupe que quelques lignes dans un traité de plus de 500 pages. La famille L'Hermite est cependant présente tout au long du traité, dans les ornements typographiques : bandeaux, culs-de-lampe et cartouches. Trois de ces ornements, utilisés de manière récurrente dans l'ouvrage, constituent des variations sur la devise " prier vaut à l'Hermite », emprunteé à l'un des ancêtres mythiques, Tristan L'Hermite. La devise est illustrée tantôt par une image de la première croisade $^{29}$, tantôt par la prise de Jérusalem : autant d'allusions à un autre ancêtre mythique des L'Hermite, Pierre l'Ermite. Ailleurs, elle est accompagnée par les armoiries de toutes les familles glorieuses dont l'auteur revendique l'alliance: Pierre l'Ermite, les dauphins d'Auvergne, les La Roche Aymon, les Miron de Barcelone et la famille de Château-Chaslons ${ }^{30}$. Ces familles fournissent la matière d'autres ornements : une image des quatre fils Aymon, qui reprend une longue tradition d'illustrations de romans de chevalerie, pour La Roche-Aymon ${ }^{31}$, un duel pour Jean de Saint-Prés ${ }^{32}$, un pénitent pardonné par un enfant-roi pour les Miron $^{33}$. En parallèle des généalogies des familles de Touraine proposées par le texte et de leurs références savantes, se déploie ainsi une généalogie de l'auteur en images, largement fantasmatique et associée à un imaginaire romanesque. Ces ornements typographiques contribuent à faire du traité généalogique un ouvrage d'agrément, mobilisant le même imaginaire chevaleresque que les romans ou les tragi-comédies, et susceptible de plaire au public mondain.

\section{Une idéologie du mérite personnel}

Cette conception de l'écriture généalogique comme écriture d'agrément va de pair avec une idéologie de la valeur qui privilégie le mérite personnel au détriment du lignage. Alors que l'on attendrait de la généalogie qu'elle légitime le pouvoir par l'inscription dans une lignée continue, les traités de Jean-Baptiste L'Hermite mobilisent de manière privilégiée une rhétorique du mérite personnel. Dans le passage qu'il consacre à André Duchesne, il affirme que son savoir et ses œuvres auraient suffi à assurer sa gloire, quelle que soit sa naissance :

\footnotetext{
Histoire généalogique de Touraine..., op. cit., p. 510-511.

Ibid., par exemple en haut de la p. 53.

Iibid., par exemple p. 52.

Ibid., par exemple p. 55.

Ibid., par exemple p. 65.

Ibid., par exemple p. 319.
} 
Quand les clartés d'une noble Naissance n'auroient pas accompagné le mérite de ce prodige de sçavoir et de penetrantes lumières, lequel sous le nom d'André du Chesne, s'est acquis tant de partisans entre les Gentilshommes, et à qui l'histoire doit tant d'éloges pour les advantages qu'elle reçoit de ses doctes connoissances, la posterité ne devroit pas avoir moins de veneration pour sa memoire et pour son sang ${ }^{34}$.

Le discours généalogique est relégué au second plan, et le texte se consacre à l'énumération des ouvrages de l'historien.

De même, Saint-Aignan se serait « fait passage au temple de l'immortalité par les armes de [son] propre mérite » même s'il « ne port[ait] pas dans [ses] veines le sang des premiers princes de l'Europe ».

C'est depuis longtemps que vous vous êtes couronné par les efforts de vostre seule valeur, et que vostre vertu soutient touste vostre fortune. Il n'y a point eu d'occasion dans nos temps, où la gloire appelle les Héros, où nous ne vous ayons remarqué entre les plus ardents et les plus advancez. ... La haute élévation de pouvoir et de gloire où le plus grand roi du monde vous a placé ; les premières dignitez de la Couronne que vous remplissez, sont aussi les justes récompenses de vos services. (n.p.)

La valeur du gentilhomme vient des services qu'il a rendus à la monarchie et pas de l'ancienneté de son lignage ${ }^{35}$. La fonction de l'écriture généalogique s'en trouve redéfinie : il ne s'agit pas tant de prouver l'ancienneté d'un lignage que de représenter un pouvoir présent de manière agréable. Elle est un instrument de la magnificence, de la construction (fantasmatique) et de l'exhibition d'une identité glorieuse.

Comme les précédents traités généalogiques de Jean-Baptiste L'Hermite, l'Histoire généalogique de Touraine paraît ainsi se situer en dehors du clivage entre histoire et fiction et affirmer la continuité entre écrits historiques, romans, pièces de théâtre et poésies. La généalogie, ici, s'écrit avec la littérature ${ }^{36}$ : non pas tant la littérature comme corpus - certes, le traité cite des romans, mais l'identification de ces textes comme « romans » et comme « littérature » est très postérieure - que la littérature comme valeur. Placé sous l'autorité d'un nom d'écrivain (le nom de Tristan), dédié à un protecteur des lettres, le traité généalogique cherche à s'approprier cette valeur nouvelle. Le processus d'institution de la littérature donne de la valeur à des écrits qui ne se réclament d'aucune autorité de savoir : il ouvre ainsi la possibilité de promouvoir une écriture généalogique agréable, indépendamment de sa vérité historique.

$* * *$

Étudier les écrits généalogiques de Jean-Baptiste L'Hermite de Soliers a pour premier effet de défaire le lien entre écriture généalogique et construction d'une histoire scientifique. Jean-Baptiste L'Hermite est un polygraphe et dans ses ouvrages, la généalogie voisine avec le théâtre, les vers héroïques ou burlesques, le roman à clé. Elle apparait alors comme une forme de l'écriture épidictique et de l'écriture de soi inscrite dans des relations de clientèle, ainsi que comme un genre à succès susceptible de plaire au public mondain.

La présence récurrente de la figure du poète Tristan L'Hermite dans les écrits généalogiques de son frère pose la question de leur caractère « littéraire ». En plaçant ses traités sous l'autorité de Tristan, Jean-Baptiste L'Hermite construit une continuité entre les tragédies et la poésie d'éloge de son frère et ses propres écrits généalogiques. Ce geste suppose l'existence d'une valeur attachée à l'écriture de Tristan, et qui peut être mobilisée pour valoriser les généalogies de Jean-Baptiste, non seulement auprès de protecteurs communs, mais bien aussi auprès d'un public plus large. En ce sens, il s'appuie sur le processus en cours d'institution de la littérature, voire y contribue. Plus que d'une

$34 \quad$ Ibid., p. 533.

35 Cette idéologie galante du mérite personnel est promue par la monarchie dans les années 1660. Sur ce sujet, je me permets de renvoyer à mon article : « Amour chevaleresque, amour galant et discours politiques de l'amour dans Les Plaisirs de l'île enchantée (1664) », Littératures classiques, 2009, Les discours artistiques de l'amour à l'âge classique, 2 (69), p.65-78.

36 Ces lignes doivent beaucoup au séminaire du GRIHL (EHESS - Université Paris 3), dans lequel se développe une réflexion sur « l'efficace ordinaire du littéraire ». 
écriture littéraire, il relève d'une écriture avec la littérature: une écriture qui s'appuie sur l'émergence de la littérature comme valeur pour fonder sa propre valeur.

C'est à la lumière de ce processus d'institution que doivent être lues les accusations dont ces traités font l'objet. En affirmant que la valeur des écrits généalogiques tient à leur vérité historique, en associant vénalité et imposture et en faisant par conséquent de la dépendance au pouvoir monarchique la condition de cette vérité historique, elles prennent position dans un débat sur les relations entre valeur des écrits et soumission au pouvoir politique. L'émergence du clivage entre histoire et fiction et la construction d'une histoire scientifique apparaissent alors comme des formes du contrôle politique des écrits par le pouvoir royal ${ }^{37}$.

Marine Roussillon

Université d'Artois, Textes et Cultures (EA 4028)

37 Sur ce processus d'institution paradoxal qui articule autonomie croissante des lettres et soumission au pouvoir, voir C. Jouhaud, op. cit. 